\title{
The Exponentiated Weibull-Power Function Distribution
}

\author{
Amal S. Hassan ${ }^{1}$, Salwa M. Assar ${ }^{2}$ \\ ${ }^{1}$ Department of Mathematical Statistics, Cairo University, Egypt. \\ ${ }^{2}$ Department of Mathematical Statistics, Cairo University, Egypt.
}

\begin{abstract}
In this article, we introduce an extension referred to as the exponentiated Weibull power function distribution based on the exponentiated Weibull-G family of distributions. The proposed model serves as an extension of the two-parameter power function distribution as well as a generalization to the Weibull power function presented by Tahir et al. (2016 a). Various mathematical properties of the subject distribution are studied. General explicit expressions for the quantile function, expansion of density and distribution functions, moments, generating function, incomplete moments, conditional moments, residual life function, mean deviation, inequality measures, Rényi and $\mathrm{q}$ - entropies, probability weighted moments and order statistics are obtained. The estimation of the model parameters is discussed using maximum likelihood method. Finally, the practical importance of the proposed distribution is examined through three real data sets. It has been concluded that the new distribution works better than other competing models.
\end{abstract}

Key words: Exponentiated Weibull-G family, Power function distribution, Moments, Order statistics, Maximum likelihood estimation.

\section{Introduction}

Power function (PF) distribution arises in several scientific fields. It is a flexible life time distribution that may offer a suitable fit to some sets of failure data. The power function distribution is a special model from the uniform distribution. Dallas (1976) showed that the power function is the inverse of Pareto distribution, that is, if has the power function then

has Pareto distribution. Meniconi and Barry (1996) mentioned in their studies that the power function distribution is preferred over exponential, lognormal and Weibull because it provides a better fit for failure data. More details on this distribution and its applications can be found in Ahsanullah and Lutful-Kabir (1974), Chang (2007) and Tavangar (2011).

The probability density function (pdf) of PF distribution is given by

$$
g(x ; \lambda, \theta)=\frac{\theta}{\lambda}\left(\frac{x}{\lambda}\right)^{\theta-1} ; \quad 0<x<\lambda, \theta>0
$$

where, $\lambda>0$ is the scale parameter and $\theta>0$ is the shape parameter. The corresponding cumulative distribution function (cdf) is given by 


$$
G(x ; \lambda, \theta)=\left(\frac{x}{\lambda}\right)^{\theta}
$$

In the last few years, new generated families of continuous distributions have attracted several statisticians to develop new models as well as provide great flexibility in modelling real data. These families are obtained by introducing one or more additional shape parameter(s) to the baseline distribution. We list some of the generated families as follows: the beta-G by Eugene et al.( 2002) and Jones (2004), gamma-G (type 1) by Zografos and Balakrishanan (2009), Kumaraswamy-G by Cordeiro and de Castro ( 2011), McDonald-G by Alexander et al. (2012), gamma-G (type 2) by Risti'c and Balakrishanan (2012), exponentiated generalized class by Cordeiro et al. (2013), transformed-transformer by Alzaatreh et al. (2013), Weibull- G by Bourguignon et al. (2014), the Kumaraswamy transmuted-G family by Afify et al. (2016), Kumaraswamy Weibull-G by Hassan and Elgarhy (2016a), exponentiated Weibull-G by Hassan and Elgarhy (2016 b) and additive Weibull-G by Hassan and Hemeda (2016), transmuted Weibull- G by Alizadeh et al. (2016), new Weibull-G by Tahir et al. (2016 b), new generalized Weibull-G by Cordeiro et al. (2015), beta Weibull-G by Yousof et al. (2017), generalized additive Weibull-G by Hassan et al. (2017) and generalized Marshall-Olkin Kumaraswamy-G family of distributions by Chakraborty and Handique (2017) among others.

Some recent extended distributions from power function have been studied by some authors. Beta power function distribution was presented by Cordeiro and Brito (2012) based on beta-G family. The Weibull power function was suggested by Tahir et al. (2016a) by using the Weibull-G family. Oguntunde et al. (2015) suggested the Kumaraswamy power distribution (KPF). Transmuted power function (TPF) was proposed by Haq et al. (2016). The exponentiated Kumaraswamy power function distribution was presented by Bursa and Kadilar (2017).

Based on exponentiated Weibull-generated $(\mathrm{EW}-\mathrm{G})$ presented by Hassan and Elgarhy (2016b), the cumulative distribution function of $(\mathrm{EW}-\mathrm{G})$ family is given by

$$
F(x)=\left[1-\exp \left(-\alpha\left[\frac{G(x)}{1-G(x)}\right]^{\rho}\right)\right]^{\alpha},
$$

where $\alpha, \beta>0$ are the two shape parameters and $\alpha>0$ is the scale parameter. The cdf (3) provides a wider family of continuous distributions. The pdf corresponding to (3) is given by

$$
f(x)=\frac{a \alpha \beta(G(x))^{\alpha-1} g(x)}{(1-G(x))^{\beta+1}} e^{-\alpha\left[\frac{G(x)}{1-G(x)}\right\}^{\rho}}\left[1-\exp \left(-\alpha\left[\frac{G(x)}{1-G(x)}\right]^{\rho}\right)\right]^{\alpha-1} .
$$

In this study, we introduce a new five-parameter model as a competitive extension for the power function distribution using the $\mathrm{EW}-\mathrm{G}$ family. The rest of the paper is outlined as follows. In Section 2, we introduce the exponentiated Weibull power function (EWPF) distribution. In Section 3, we derive a very useful representation for the EWPF density and distribution functions. In the same section, some general mathematical properties of the proposed distribution are derived. The maximum likelihood method is used to drive the estimates of the model parameters in Section 4. Simulation study is performed to obtain the 
maximum likelihood estimates of the model paramerets in Section 5. Applicability of the proposed model is shown and compared with other competing probability models in Sestion 6. At the end, concluding remarks are outlined.

\section{The Exponentiated Weibull-Power Distribution} family.

In this section, the five-parameter EWPF distribution is obtained based on the EW-G

The cdf of the exponentiated Weibull power function distribution, denoted by $\operatorname{EWPF}(a, \alpha, \beta, \lambda, \theta)$ is obtained by inserting the $\operatorname{cdf}(2)$ in cdf (3) as follows

$$
F(x ; \Psi)=\left[1-\exp \left(-\alpha\left(\frac{x^{\theta}}{\lambda^{\theta}-x^{\theta}}\right)^{\beta}\right)\right]^{3} ; \quad a, \alpha, \beta, \lambda, \theta>0 \quad, 0<x<\lambda,
$$

where, $\Psi \equiv(a, \alpha, \beta, \lambda, \theta)$, is the set of parameters. The pdf of EWPF distribution is obtained by inserting the pdf (1) and cdf (2) into (4) as the following

$$
f(x ; \Psi)=\frac{a \alpha \beta \theta \lambda^{\theta} x^{\theta \beta-1}}{\left(\lambda^{\theta}-x^{\theta}\right)^{\theta+1}} \exp \left(-\alpha\left(\frac{x^{\theta}}{\lambda^{\theta}-x^{\theta}}\right)^{\theta}\right)\left[1-\exp \left(-\alpha\left(\frac{x^{\theta}}{\lambda^{\theta}-x^{\theta}}\right)^{\theta}\right)\right]^{\theta-1} \text {. }
$$

Note that; for $a=1$, the pdf (6) reduces to Weibull power function which is carried out by Tahir et al. (2016a). The survival, hazard rate, reversed-hazard rate and cumulative hazard rate functions of EWPF distribution are respectively given by

$$
\begin{aligned}
& R(x ; \Psi)=1-\left[1-\exp \left(-\alpha\left(\frac{x^{\theta}}{\lambda^{\theta}-x^{\theta}}\right)^{\beta}\right)\right]^{\alpha},
\end{aligned}
$$

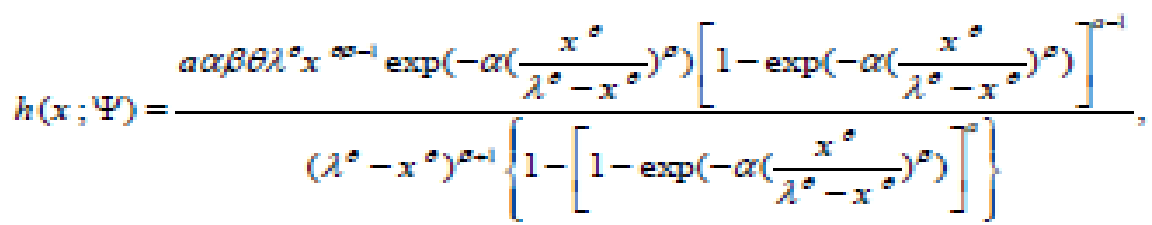

$$
\begin{aligned}
& \tau(x ; \Psi)=\frac{a \alpha \beta \theta \lambda^{\theta} x^{\theta \sigma-1} \exp \left(-\alpha\left(\frac{x^{\theta}}{\lambda^{\theta}-x^{\theta}}\right)^{\mathcal{\theta}}\right)}{\left(\lambda^{\theta}-x^{\theta}\right)^{\theta+1}\left[1-\exp \left(-\alpha\left(\frac{x^{\theta}}{\lambda^{\theta}-x^{\theta}}\right)^{\mathcal{\theta}}\right)\right]},
\end{aligned}
$$

and

$$
H\left(x ; \Psi^{\prime}\right)=-\ln \left|R\left(x ; \Psi^{\prime}\right)\right|=-\ln \left|1-\left[1-\exp \left(-\alpha\left(\frac{x^{\theta}}{\lambda^{\theta}-x^{\theta}}\right)^{\sigma}\right)\right]^{\alpha}\right| .
$$

Plots of the pdf and hazard rate function (hrf) of the EWPF are displayed in Figures 1 and 2 for selected parameter values. It is clear from Figure 1 that the EWPF densities take various 
shapes such as symmetrical, left-skewed, reversed-J, right skewed and unimodal. Also, Figure 2 indicates that EWPF hrfs can have increasing, decreasing, constant and U-shaped. This fact implies that the EWPF can be very useful for fitting data sets with various shapes.

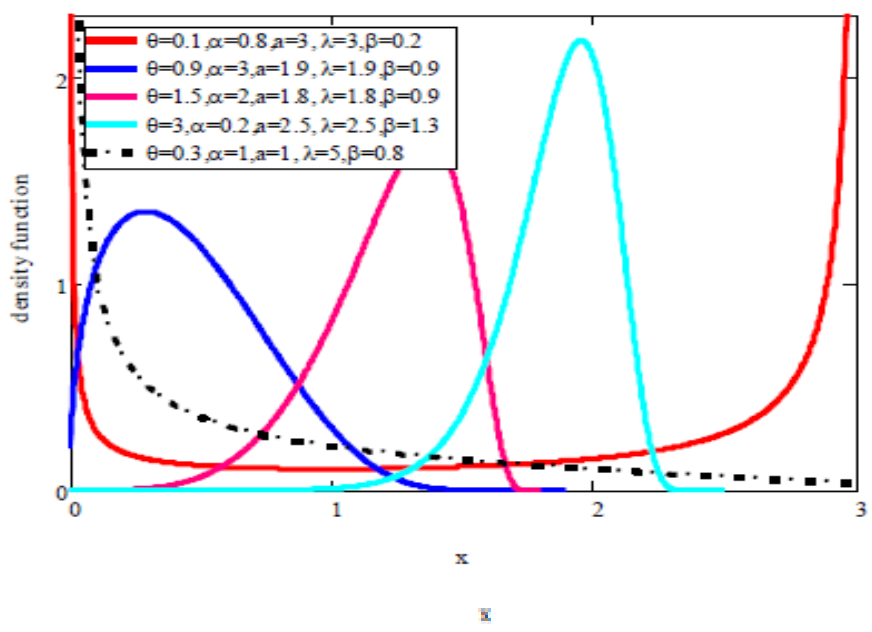

Figure 1 Plots of the pdf for some parameters

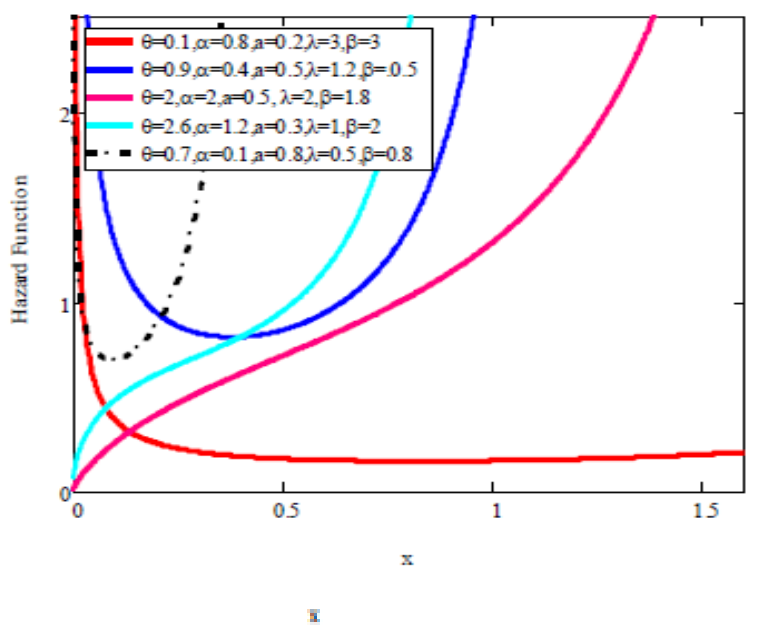

Figure 2 Plots of the hrf for some parameters

\section{Statistical Properties}

Here, we provide some properties of the EWPF distribution.

\subsection{Important Expansions}

In this subection, two usefuel representations of the pdf and cdf for exponentiated Weibull power function distribution are derived.

The pdf (6) can be rewritten as follows

$$
f \quad f(x ; \Psi)=\frac{a \alpha \beta \theta}{\lambda\left(1-\left(\frac{x}{\lambda}\right)^{\theta}\right)^{\beta+1}}\left(\frac{x}{\lambda}\right)^{\theta \beta-1} e^{-\alpha\left[\frac{\left(\frac{x}{\lambda}\right)^{\theta}}{1-\left(\frac{x}{\lambda}\right)^{\beta}}\right]}\left[1-\exp \left(-\alpha\left[\frac{\left(\frac{x}{\lambda}\right)^{\theta}}{1-\left(\frac{x}{\lambda}\right)^{\theta}}\right]^{\beta}\right)\right]^{\alpha-1} .
$$

The generalized binomial theorem, for $\beta>0$ is real non integer and $|Z|<1$ is given by

$$
(1-z)^{\mathcal{\beta}-1}=\sum_{i=0}^{\infty}(-1)^{i}\left(\begin{array}{c}
\mathcal{\beta}-1 \\
i
\end{array}\right) z^{i} \text {. }
$$

Then, by applying the binomial theorem (8) in (7), the probability density function of EWPF distribution where $a$ is a real non integer becomes 


$$
f(x ; \Psi)=\sum_{j=0}^{\infty}(-1)^{j}\left(\begin{array}{c}
a-1 \\
j
\end{array}\right) \frac{a \alpha \beta \theta}{\lambda\left(1-\left(\frac{x}{\lambda}\right)^{\theta}\right)^{\beta+1}}\left(\frac{x}{\lambda}\right)^{\theta \beta-1} \exp \left(-\alpha(j+1)\left[\frac{\left(\frac{x}{\lambda}\right)^{\theta}}{1-\left(\frac{x}{\lambda}\right)^{\theta}}\right]^{\beta}\right) .
$$

By using the power series for the exponential function and the following binomial expansion

$$
(1-z)^{-k}=\sum_{j=0}^{\infty} \frac{\Gamma(k+j) z^{j}}{\Gamma(k) j !}, \quad|z|<1, k>0 .
$$

Hence the pdf of the EWPF distribution takes the following form

$$
\begin{aligned}
f\left(x ; \Psi^{\prime}\right) & =\sum_{i, j, k-0}^{\infty} w_{i, j, k} g_{\theta(\beta+\beta i+k)}(x), \\
w_{i, j, k} & =(-1)^{j+i}\left(\begin{array}{c}
a-1 \\
j
\end{array}\right) \frac{a \alpha \beta(\alpha(j+1))^{i} \Gamma(\beta+\beta i+k)}{k ! i ! \Gamma(\beta+\beta i+1)},
\end{aligned}
$$

Where $g_{\theta\left(\beta+\beta_{i}+k\right)}(x)$ denotes the pdf of PF distribution with parameters $\theta\left(\beta+\beta_{i}+\mathrm{k}\right)$ and $\lambda$.

Furtheremore, an expansion for the cumulative distribution function is derived. Using binomial expansion for $(\mathrm{F}(\mathrm{x} ; \Psi))^{\mathrm{s}}$, where $\mathrm{s}$ is an integer and $a$ is a real non integer, leads to:

$$
\left(F\left(x ; \Psi^{s}\right)\right)^{s}=\sum_{p=0}^{\infty}(-1)^{p}\left(\begin{array}{c}
a s \\
p
\end{array}\right) \exp \left(-\alpha p\left[\frac{\left(\frac{x}{\lambda}\right)^{\theta}}{1-\left(\frac{x}{\lambda}\right)^{\theta}}\right]^{\beta}\right) .
$$

Applying the power series for the exponential function and the relation (10) where $\beta$ is real non integer, then the previous cdf takes the following form

$$
\left(F\left(x ; \Psi^{s}\right)\right)^{s}=\sum_{p, m=0}^{\infty}(-1)^{p+q}\left(\begin{array}{c}
a s \\
p
\end{array}\right) \frac{(\alpha p)^{q} \Gamma(m+\beta q)}{\Gamma(\beta q) m !}\left[\left(\frac{x}{\lambda}\right)\right]^{\theta(\beta q+m)} .
$$

Or, it can also be written as,

$$
\begin{aligned}
(F(x ; \Psi))^{s} & =\sum_{p, q, m=0}^{\infty} \eta_{p, q, m} G_{\theta(\beta q+m)}(x), \\
\eta_{p, q, m} & =(-1)^{p+q}\left(\begin{array}{c}
a s \\
p
\end{array}\right) \frac{(\alpha p)^{q} \Gamma(m+\beta q)}{\Gamma(\beta q) m !} .
\end{aligned}
$$

\subsection{Quantile Function} follows

The quantile function, say $\mathrm{x}=\mathrm{Q}(\mathrm{u})=F^{-1}(u)$ of $\mathrm{X}$ can be obtained by inverting (5) as 


$$
x=Q(u)=\lambda\left[1+\left\{\frac{-1}{\alpha} \ln \left(1-u^{\frac{1}{a}}\right)\right\}^{\frac{-1}{\beta}}\right]^{\frac{-1}{\theta}},
$$

where, $\mathrm{u}$ is a uniform random variable on the unit interval $(0,1)$. In particular, the first quartile, median and third quartile are obtained by subsituting $\mathrm{u}=0.25,0.5$ and $\mathrm{u}=0.75$ in (13).

The Bowley skewness introduced by Kenney and Keeping (1962) based on quartiles is given by

$$
B=\frac{Q\left(\frac{3}{4}\right)-2 Q\left(\frac{1}{2}\right)+Q\left(\frac{1}{4}\right)}{Q\left(\frac{3}{4}\right)-Q\left(\frac{1}{4}\right)} .
$$

The Moors kurtosis (see Moors (1988)) based on octiles is given by

$$
M=\frac{Q\left(\frac{7}{8}\right)-Q\left(\frac{5}{8}\right)+Q\left(\frac{3}{8}\right)-Q\left(\frac{1}{8}\right)}{Q\left(\frac{6}{8}\right)-Q\left(\frac{2}{8}\right)},
$$

where $\mathrm{Q}($.) denotes the quantile function. Plots of the skewness and kurtosis for some choices of $a$ as function of $\beta$ are shown in Figure 3. The plots indicate the variability of these measures on the shape parameters.

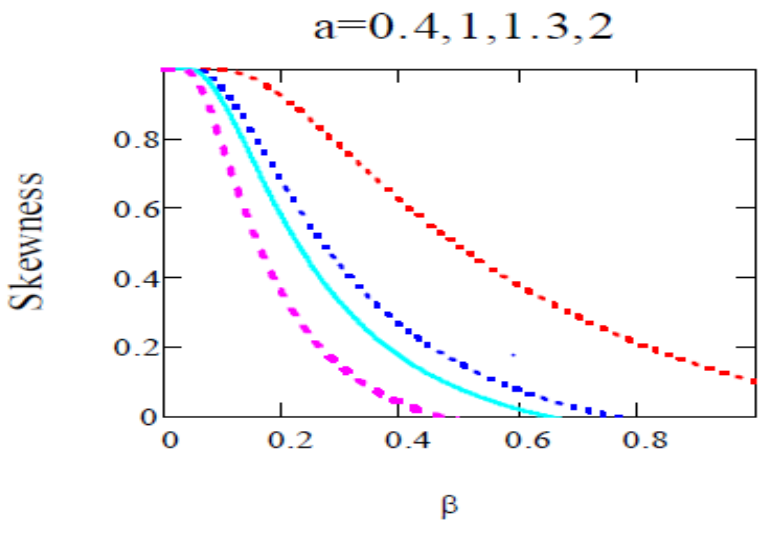

(i)

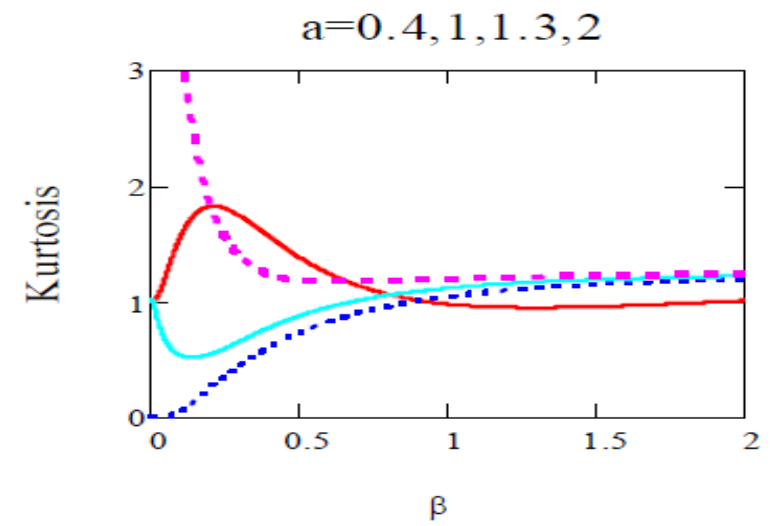

(ii)

Figure 3: Skewness (i) and kurtosis (ii) plots for EWPF distribution based on quantile function

\subsection{Moments}

This subsection concerns with the rth moment and the moment generating function for EWPF distribution. 
If $\mathrm{X}$ has the pdf (11), then its rth moment can be obtained as follows

$$
\mu_{r}^{\prime}=E\left(\mathrm{X}^{r}\right)=\int_{-\infty}^{\infty} x^{r} f(x ; \Psi) d x
$$

Inserting (11) into (14) yields:

$$
\begin{gathered}
\mu_{r}^{\prime}=E\left(X^{r}\right)=\sum_{i, j, k=0}^{\infty} w_{i, j, k} \int_{0}^{\lambda} x^{r} g_{\theta(\beta+\beta i+k)}(x) d x . \\
\mu_{r}=\sum_{i, j, k=0}^{\infty} w_{i, j, k} \frac{\lambda^{r} \theta(\beta+\beta i+k)}{r+\theta(\beta+\beta i+k)}, \quad r=1,2, \ldots
\end{gathered}
$$

Setting $r=1,2,3,4$ in (15), we can obtain the first four moments about zero. Generally, the moment generating function of EWPF distribution is obtained through the following relation

$$
M_{X}(t)=\sum_{r=0}^{\infty} \frac{t^{r}}{r !} E\left(X^{r}\right)=\sum_{i, j, k=0}^{\infty} w_{i, j, k} \frac{t^{r}}{r !} \frac{\lambda^{r} \theta(\beta+\beta i+k)}{r+\theta(\beta+\beta i+k)} .
$$

\subsection{Incomplete and Conditional Moments}

The main application of the first incomplete moment refers to the Bonferroni and Lorenz curves. These curves are very useful in economics, reliability, demography, insurance and medicine. The incomplete moments, say $\varphi_{s}(t)$, is given by

$$
\varphi_{s}(t)=\int_{0}^{t} x^{s} f(x ; \Psi) d x \text {. }
$$

Using (11), then $\varphi_{S}(t)$ can be written as follows

$$
\varphi_{s}(t)=\sum_{i, j, k=0}^{\infty} w_{i, j, k} \frac{\theta(\beta+\beta i+k) t^{s+\theta(\beta+\beta i+k)}}{\lambda^{\theta(\beta+\beta i+k)}(s+\theta(\beta+\beta i+k))}
$$

Further, the conditional moments, say $r_{s}(t)$, is given by

$$
\gamma_{s}(t)=\int_{t}^{\infty} x^{s} f(x ; \Psi) d x
$$

Hence, by using pdf (11), we can write

$$
\gamma_{s}(t)=\sum_{i, j, k=0}^{\infty} w_{i, j, k} \frac{\theta(\beta+\beta i+k)}{\lambda^{\theta(\beta+\beta i+k)}}\left[\frac{\lambda^{s+\theta(\beta+\beta i+k)}-t^{s+\theta(\beta+\beta i+k)}}{(s+\theta(\beta+\beta i+k))}\right] .
$$

Additionally, the mean deviation can be calculated by the following relation

$$
\mathcal{S}_{1}(X)=2 \mu F(\mu)-2 T(\mu) \quad \text { and } \quad \mathcal{S}_{2}(X)=\mu-2 T(M) \text {, }
$$




\section{The Exponentiated Weibull-Power Function Distribution}

where, $\mathrm{T}(\mathrm{q})=\int_{0}^{q} x f(x) d x$ which is the first incomplete moment. 
By using (11) then,

$$
\begin{gathered}
T(\mu)=\int_{0}^{\mu} x f(x) d x=\sum_{i, j, k=0}^{\infty} w_{i, j, k} \frac{\theta(\beta+\beta i+k) \mu^{\theta(\beta+\beta i+k)+1}}{\lambda^{\theta(\beta+\beta i+k)}(\theta(\beta+\beta i+k)+1)}, \\
T(M)=\int_{0}^{M} x f(x) d x=\sum_{i, j, k=0}^{\infty} w_{i, j, k} \frac{\theta(\beta+\beta i+k) M^{\theta(\beta+\beta i+k)+1}}{\lambda^{\theta(\beta+\beta i+k)}(\theta(\beta+\beta i+k)+1)} .
\end{gathered}
$$

\subsection{Residual Life Function}

The residual life plays an important role in life testing situations and reliability theory. The nth moment of the residual life is given by:

$$
m_{n}(t)=E\left[(X-t)^{n} \mid X>t\right]=\frac{1}{R(t)} \int_{t}^{\infty}(x-t)^{n} f(x) d x .
$$

The nth moment of the residual life of EWPF random variable is obtained by inserting pdf (11) in (16) as follows

$$
m_{n}(t)=\frac{1}{R(t ; \Psi)} \sum_{r=0}^{n} \sum_{i, j, k}^{\infty}(-1)^{n-r} w_{i, j, k}\left(\begin{array}{c}
n \\
r
\end{array}\right) t^{n-r} \int_{t}^{\lambda} x^{r} g_{\theta(\beta+\beta i+k)}(x) d x
$$

So, the nth moment of the residual life of EWPF can be represented as follows

$$
m_{n}(t)=\frac{\sum_{r=0}^{n} \sum_{i, j, k}^{\infty}(-1)^{n-r} w_{i, j, k}\left(\begin{array}{l}
n \\
r
\end{array}\right) t^{n-r} \frac{\theta(\beta+\beta i+k)}{\lambda^{\theta(\beta+\beta i+k)}}\left[\frac{\lambda^{r+\theta(\beta+\beta i+k)}-t^{r+\theta(\beta+\beta i+k)}}{r+\theta(\beta+\beta i+k)}\right]}{\left[1-\left[1-\exp \left(-\alpha\left(\frac{t^{\theta}}{\lambda^{\theta}-t^{\theta}}\right)^{\mathcal{\beta}}\right)\right]^{\alpha}\right]}
$$

Another interesting function is the mean residual life (MRL) function or the life expectation at age $\mathrm{x}$ defined by $m_{1}(t)=[(X-t) \mid X>t]$, which represents the expected additional life length for a unit which is alive at age $\mathrm{x}$ The MRL of the EWPF distribution can be obtained by setting $\mathrm{n}=1$ in (17).

\subsection{Inequality Measures}

Lorenz and Bonferroni curves are the most widely used inequality measures in income and wealth distribution. Zenga curve was presented by Zenga (2007). Here, the Lorenz, Bonferroni and Zenga curves for the EWPF distribution are derived. The Lorenz, Bonferroni and Zenga curves are obtained, respectively, as follows 


$$
\begin{gathered}
L_{F}(t)=\frac{\int_{0}^{t} x f(x) d x}{E(X)}=\frac{\sum_{i, j, k=0}^{\infty} w_{i, j, k} \frac{\theta(\beta+\beta i+k) t^{\theta(\beta+\beta i+k)+1}}{\lambda^{\theta(\beta+\beta i+k)}(\theta(\beta+\beta i+k)+1)}}{\sum_{i, j, k=0}^{\infty} w_{i, j, k} \frac{\lambda \theta(\beta+\beta i+k)}{\theta(\beta+\beta i+k)+1}} . \\
B_{F}(t)=\frac{\int_{0}^{t} x f(x) d x}{E(X) F(t)}=\frac{L_{F}(t)}{F(t)}=\frac{\sum_{i, j, k-0}^{\infty} w_{i, j, k} \frac{\theta(\beta+\beta i+k) t^{\theta(\beta+\beta i+k)+1}}{\lambda^{\theta(\beta+\beta i+k)}(\theta(\beta+\beta i+k)+1)}}{\left[1-\exp \left(-\alpha\left(\frac{t^{\theta}}{\lambda^{\theta}-t^{\theta}}\right)^{\beta}\right)\right]_{i, j, k-0}^{a} w_{i, j, k}^{\infty} \frac{\lambda \theta(\beta+\beta i+k)}{\theta(\beta+\beta i+k)+1}} .
\end{gathered}
$$

and

$$
A_{F}(t)=1-\frac{\mu^{-}(t)}{\mu^{+}(t)}
$$

where

$$
\mu^{-}(t)=\frac{\int_{0}^{t} x f(x) d x}{E(X)}=\frac{\sum_{i, j, k=0}^{\infty} w_{i, j, k} \frac{\theta(\beta+\beta i+k) t^{\theta(\beta+\beta i+k)+1}}{\lambda^{\theta(\beta+\beta i+k)}(\theta(\beta+\beta i+k)+1)}}{\sum_{i, j, k=0}^{\infty} w_{i, j, k} \frac{\lambda \theta(\beta+\beta i+k)}{\theta(\beta+\beta i+k)+1}} .
$$

And

$$
\mu^{+}(t)=\frac{\int_{t}^{\infty} x f(x) d x}{1-F(t)}=\frac{\sum_{i, j, k=0}^{\infty} w_{i, j, k} \frac{\theta(\beta+\beta i+k)}{\lambda^{\theta(\beta+\beta i+k)}}\left[\frac{\lambda^{1+\theta(\beta+\beta i+k)}-t^{1+\theta(\beta+\beta i+k)}}{(1+\theta(\beta+\beta i+k))}\right]}{\left[1-\left[1-\exp \left(-\alpha\left(\frac{t^{\theta}}{\lambda^{\theta}-t^{\theta}}\right)^{\beta}\right)\right]^{\alpha}\right]} .
$$

\subsection{Rényi and q - Entropies}

The entropy of a random variable $\mathrm{X}$ is a measure of variation of uncertainty and has been used in many fields such as physics, engineering and economics. The Rényi entropy is defined by

$$
I_{\mathcal{S}}(X)=\frac{1}{1-\delta} \log \int_{-\infty}^{\infty} f(x)^{\mathcal{s}} d x, \quad \delta>0 \text { and } \delta \neq 1 .
$$

By applying the binomial theory (8), (10) and exponential expansion, then the pdf $f(x ; \Psi)^{s}$ can be expressed as follows

$$
(f(x ; \Psi))^{\mathcal{S}}=\sum_{i, j, k=0}^{\infty} \xi_{i, j, k}\left(\frac{x}{\lambda}\right)^{\mathcal{\delta}(\theta \beta-1)+\theta \beta i+\theta k} .
$$

where 
$\xi_{i, j, k}=(-1)^{i+j}\left(\begin{array}{c}\delta(a-1) \\ i\end{array}\right) \frac{(\alpha(\delta+i))^{j}(a \alpha \beta \theta)^{\mathcal{S}} \Gamma(\delta(\beta+1)+\beta i+k)}{j ! \lambda^{\mathcal{S}} \Gamma(\delta(\beta+1)+\beta i) k !}$.

Therefore, the Rényi entropy of EWPF distribution is given by

$$
I_{\mathcal{\delta}}(X)=\frac{1}{1-\delta} \log \left[\sum_{i, j, k=0}^{\infty} \frac{\xi_{i, j, k} \lambda}{(\delta(\theta \beta-1)+\theta \beta i+\theta k+1)}\right] .
$$

The q- entropy is defined by

$$
H_{q}(X)=\frac{1}{1-q} \log \left(1-\int_{-\infty}^{\infty} f(x ; \Psi)^{q} d x\right), q>0 \text { and } q \neq 1 .
$$

Therefore, the q- entropy of distribution is given by

$$
H_{q}(X)=\frac{1}{1-q} \log \left\{1-\left[\sum_{i, j, k=0}^{\infty} \frac{\xi_{i, j, k} \lambda}{(q(\theta \beta-1)+\theta \beta i+\theta k+1)}\right]\right\} \text {. }
$$

\subsection{The Probability Weighted Moments}

A general theory of probability weighted moments (PWMs) was initially introduced by Greenwood et al. (1979) to derive estimators of the parameters and quantiles of generalized distributions.

The probability weighted moments of a random variable $\mathrm{X}$, say $\tau_{r, s}$ is formally defined by

$$
\tau_{r, s}=E\left[X^{r} \mathrm{~F}(x)^{s}\right]=\int_{-\infty}^{\infty} x^{r} \mathrm{f}(x)(\mathrm{F}(x))^{s} d x
$$

Therefore, PWM of the EWPF distribution is obtained by inserting (11) and (12) into (18), as follows

$$
\tau_{r, s}=\sum_{i, j, k=0}^{\infty} w_{i, j, k} \sum_{p, q, m=0}^{\infty} \eta_{p, q, m} \int_{0}^{\lambda} \frac{\theta(\beta+\beta i+k)}{\lambda}\left(\frac{x}{\lambda}\right)^{\theta(\beta+\beta i+k+\beta q+m)-1} d x .
$$
form

Hence, the PWM of exponentiated Weibull power function distribution takes the following

$$
\tau_{r, s}=\sum_{i, j, k=0}^{\infty} \sum_{p, q, m=0}^{\infty} \frac{\eta_{p, q, m} w_{i, j, k}(\beta+\beta i+k)}{(\beta+\beta i+k+\beta q+m)}
$$

\subsection{Order Statistics}

Let $X_{1: n}<X_{2: n}<\cdots<X_{n: n}$ be the order statistics of a random sample of size $\mathrm{n}$ following the EWPF, the pdf of the rth order statistic is given by

$$
f_{r: n}(x)=\frac{f(x)}{B(r, n-r+1)} \sum_{v=0}^{n-r}(-1)^{v}\left(\begin{array}{c}
n-r \\
v
\end{array}\right) F(x)^{r+v-1},
$$


where, $\mathrm{B}(.,$.$) is the beta function. Inserting (11) and (12) into (19) by replacing s with \mathrm{v}+$ $\mathrm{r}-1$ leads to

$$
\begin{aligned}
f_{r, n}(x)= & \frac{1}{B(r, n-r+1)} \sum_{v=0 i, j, k, p, q, m=0}^{n-r} \sum_{i, j, k, p, q, m}^{\infty} \frac{\theta(\beta+\beta i+k)}{\lambda}\left(\frac{x}{\lambda}\right)^{\theta(\beta+\beta i+k+\beta q+m)-1,}, \\
& \mathrm{~K}_{i, j, k, p, q, m}=(-1)^{v+p+q} w_{i, j, k}\left(\begin{array}{c}
n-r \\
v
\end{array}\right)\left(\begin{array}{l}
a(v+r-1) \\
p
\end{array}\right) \frac{(\alpha p)^{q} \Gamma(m+\beta q)}{\Gamma(\beta q) m !} .
\end{aligned}
$$

In particuler, the the pdf of the smallest order statistic is obtained by substituting $r=1$ in (20) as follows

$$
\begin{gathered}
f_{1-n}=n \sum_{v=0}^{n-1} \sum_{i, j, p, q, m=0}^{\infty} \pi_{i, j, k, p, q, m} \frac{\theta(\beta+\beta i+1)}{\lambda}\left(\frac{x}{\lambda}\right)^{\theta(\beta+\beta i+k+\beta q+m)-1} \\
\pi_{i, j, k, p, q, m}=(-1)^{v+p+q}\left(\begin{array}{c}
n-1 \\
v
\end{array}\right) w_{i, j, k}\left(\begin{array}{l}
a v \\
p
\end{array}\right) \frac{(\alpha p)^{q} \Gamma(m+\beta q)}{\Gamma(\beta q) m !}
\end{gathered}
$$

Further, the the pdf of the largest order statistic is obtained by subsituting in (20) as follows

$$
\begin{aligned}
& f_{1: n}=n \sum_{i, j, p, q, m=0}^{\infty} \varpi_{i, j, k, p, q, m} \frac{\theta(\beta+\beta i+1)}{\lambda}\left(\frac{x}{\lambda}\right)^{\theta(\beta+\beta i+k+\beta q+m)-1}, \\
& \varpi_{i, j, k, p, q, m}=(-1)^{p+q} w_{i, j, k}\left(\begin{array}{c}
a v \\
p
\end{array}\right) \frac{(\alpha p)^{q} \Gamma(m+\beta q)}{\Gamma(\beta q) m !} .
\end{aligned}
$$

\section{Maximum Likelihood Estimation}

The maximum likelihood estimators of the unknown parameters for the exponentiated Weibull power function distribution are determined based on complete samples. Let $X_{1}, \ldots, X_{n}$ be observed values from the EWPF distribution with set of parameters $\Psi \equiv(a, \alpha, \beta, \lambda, \theta)^{T}$. The total log-likelihood function for the vector of parameters $\Psi$ can be expressed as

$$
\begin{aligned}
\ln L\left(\Psi^{\prime}\right)= & n \ln a+n \ln \alpha+n \ln \beta+n \ln \theta+n \theta \ln \lambda+(\theta \beta-1) \sum_{i=1}^{n} \ln x_{i}-(\beta+1) \sum_{i=1}^{n} \ln \left(\lambda^{\theta}-x_{i}{ }^{\theta}\right) \\
& -\alpha \sum_{i=1}^{n} z_{i}{ }^{\beta}+(a-1) \sum_{i=1}^{n} \ln \left[1-\exp \left(-\alpha z_{i}{ }^{\beta}\right)\right],
\end{aligned}
$$

where, $Z_{i}=\left(\frac{x_{i}^{\theta}}{\lambda^{\theta}-x_{i}^{\theta}}\right)$. The $\lambda$ is known and we estimate it from the sample maxima. The elements of the score function $\mathrm{U}(\Psi)=\left(U_{a}, U_{\alpha}, U_{\beta}, U_{\theta}\right)$ are given by

$$
U_{a}=\frac{n}{a}+\sum_{i=1}^{n} \ln \left[1-\exp \left(-\alpha z_{i}^{\beta}\right)\right] \text {, }
$$




$$
\begin{gathered}
U_{\alpha}=\frac{n}{\alpha}-\sum_{i=1}^{n} z_{i}{ }^{\beta}+(a-1) \sum_{i=1}^{n} \frac{\left.z_{i}{ }^{\beta} \exp \left(-\alpha z_{i}{ }^{\beta}\right)\right]}{\left[1-\exp \left(-\alpha z_{i}{ }^{\beta}\right)\right]} \\
U_{\beta}=\frac{n}{\beta}+\theta \sum_{i=1}^{n} \ln x_{i}-\sum_{i=1}^{n} \ln \left(\lambda^{\theta}-x_{i}{ }^{\theta}\right)-\alpha \sum_{i=1}^{n} z_{i}{ }^{\beta} \ln z_{i}+\alpha(a-1) \sum_{i=1}^{n} \frac{z_{i}{ }^{\beta} \exp \left[-\alpha z_{i}{ }^{\beta}\right] \ln z_{i}}{1-\exp \left[-\alpha z_{i}{ }^{\beta}\right]},
\end{gathered}
$$

and

$$
\begin{aligned}
U_{\theta}= & \frac{n}{\theta}+n \ln \lambda+\beta \sum_{i=1}^{n} \ln x_{i}-(\beta+1) \sum_{i=1}^{n} \frac{\lambda^{\theta} \ln \lambda-x_{i}^{\theta} \ln x_{i}}{\left(\lambda^{\theta}-x_{i}^{\theta}\right)}-\alpha \beta \sum_{i=1}^{n} z_{i}^{\beta-1}\left[\frac{\lambda^{\theta} x_{i}^{\theta}\left(\ln x_{i}-\ln \lambda\right)}{\left(\lambda^{\theta}-x_{i}^{\theta}\right)^{2}}\right] \\
& +(a-1) \sum_{i=1}^{n} \frac{\alpha \beta z_{i}^{\beta-1} \exp \left(-\alpha z_{i}^{\beta}\right)}{\left[1-\exp \left(-\alpha z_{i}^{\beta}\right)\right]}\left[\frac{\lambda^{\theta} x_{i}^{\theta}\left(\ln x_{i}-\ln \lambda\right)}{\left(\lambda^{\theta}-x_{i}^{\theta}\right)^{2}}\right] .
\end{aligned}
$$

Then the maximum likelihood estimates (MLEs) of the parameters a, $\alpha, \beta$ and $\theta$ are obtained by setting equations (21 -24) to be zero and solving them numerically.

\section{Simulation Study}

In this section, a simulation study is carried out to evaluate the performance of the MLEs of the EWPF parameters with respect to sample size. The numerical procedures are described through the following algorithm.

Step(1): A random sample $X_{1}, \ldots, X_{n}$ of sizes $\mathrm{n}=(10,20,30,50,100,200)$ are selected, these random samples are generated from the EWPF distribution.

Step(2): Assume that $\lambda$ to be known and we will take it to be one in all experiments. Eight selected parameter combinations are considered as follows: set I $(\alpha=0.5, \beta=1, \theta=1, \mathrm{a}=0.5)$, set II $(\alpha=1.5, \beta=1, \theta=1.5, \mathrm{a}=0.5)$, set III $(\alpha=0.5, \beta=0.5, \theta=1.5, \mathrm{a}=0.5)$, set IV $(\alpha=1.5$, $\beta=0.5, \theta=0.5 \mathrm{a}=1)$, set $\mathrm{V}(\alpha=0.5, \beta=0.5, \theta=0.5$, $\mathrm{a}=0.5)$, set VI $(\alpha=0.5, \beta=0.5, \theta=0.5$, $\mathrm{a}=1.5)$, set VII $(\alpha=0.5, \beta=0.5, \theta=0.5$, $\mathrm{a}=1.5)$, set VIII $(\alpha=0.5, \beta=0.5, \theta=1, \mathrm{a}=1.5)$.

Step (3): For each model parameters and for each sample size, the MLEs of $\alpha, \beta, \theta$ and a are computed.

Step (4): Steps from 1 to 3 are repeated 1000 times for each sample size and for selected sets of parameters. Then, the MLEs of the parameters, their biases and standard errors (SE) are computed.

From simulation results, the following observations can be made:

1. The standard errors for each parameter generally decrease as the sample size increases for all set of parameters as shown in Figure (4).

2. The SEs of $\alpha$ for set I have the smallest values corresponding to the other set of parameters (see Figure 4(i)), the SEs of $\beta$ for set VI have the smallest values corresponding to the other set of parameters (see Figure 4(ii)). Also, the SEs of $\theta$ for set II have the smallest values corresponding to the other set of parameters (see Figure 4(iii)), the SEs of a for set IV have the smallest values corresponding to the other set of parameters (see Figure 4(iv)). 
$\alpha$

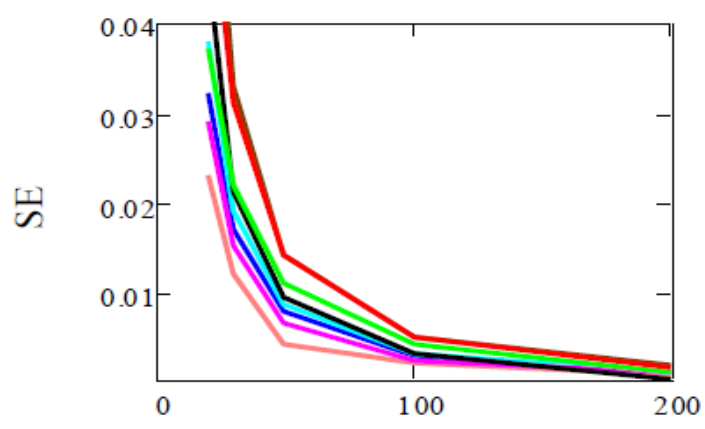

11

(i)

$\theta$

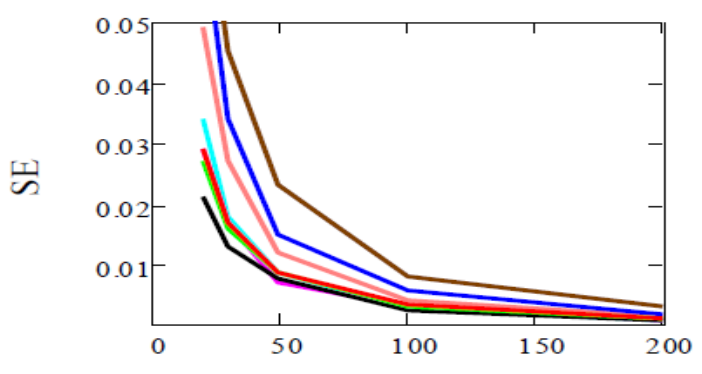

n

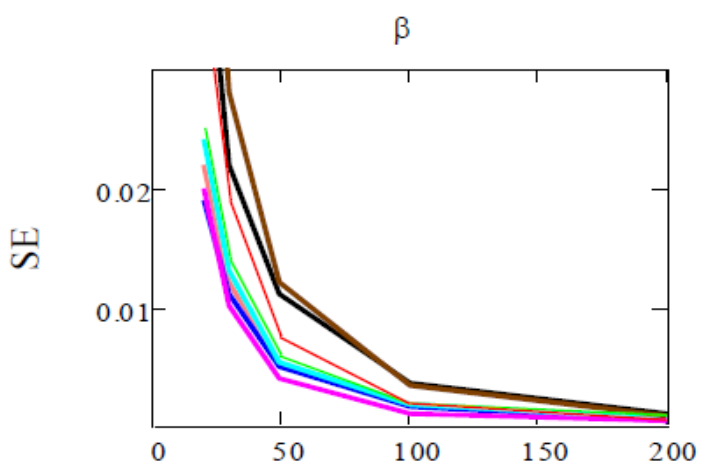

11

(ii)

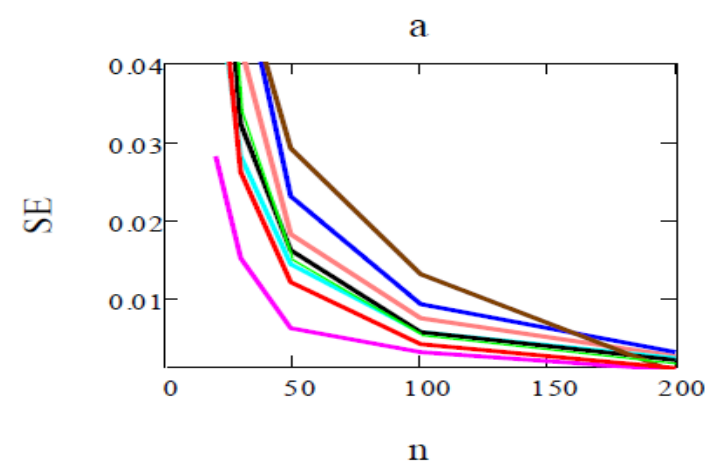

(iv)

(iii)

Figure 4: Standard errors of $\alpha, \beta, \theta$ and a for eight set of parameters

3. Most of the estimates for different parameters are positively and negatively biased which indicates that the estimates are overestimate and underestimates respectively as shown in Figure 5. Generally, the biases for each parameter decrease as the sample size increases expect for few cases (see Figure 5). 
$\alpha$

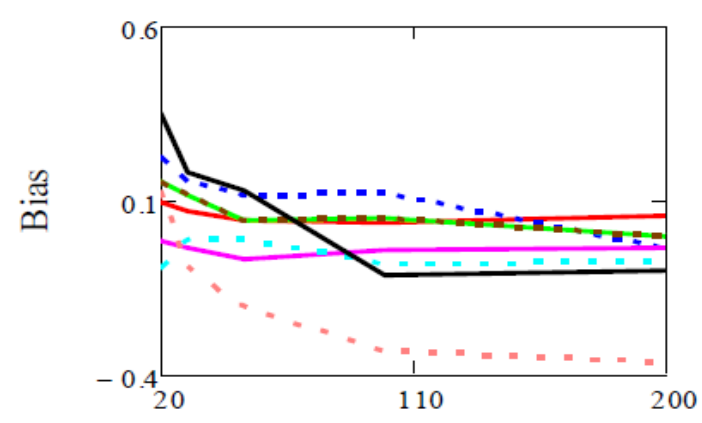

$\mathrm{n}$

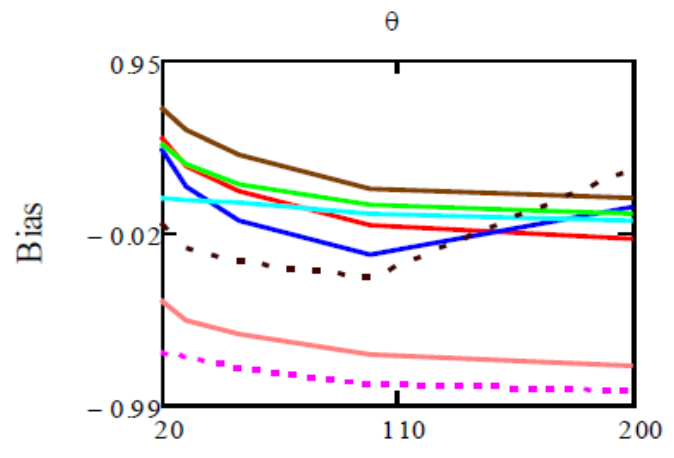

n

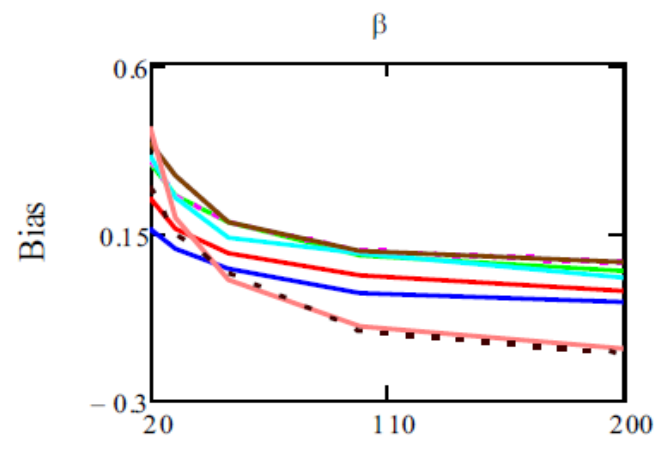

$\mathrm{n}$

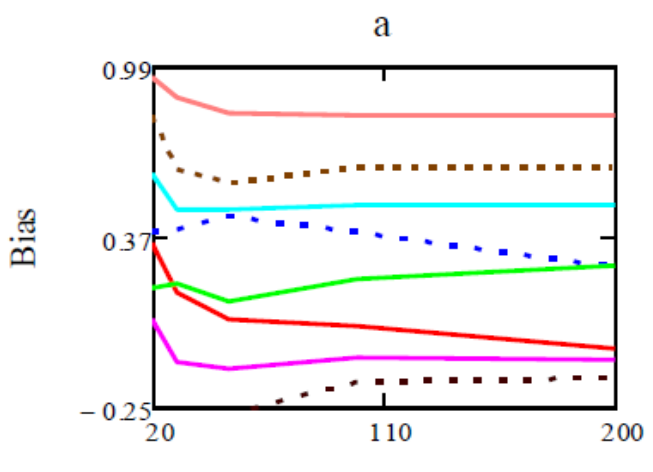

n

Figure 5: Biases of $\alpha, \beta, \theta$ and a for eight set of parameters

4. The SEs for $\beta$ in sets III, V, VI, VII and VIII take the smallest values compared to the corresponding SEs for $\theta, \alpha$, a ; while the SEs for $\theta$ in sets I, II, IV take the smallest values compared to the corresponding SEs for $\beta, \alpha$, a (see Figure 6 as particular cases). 

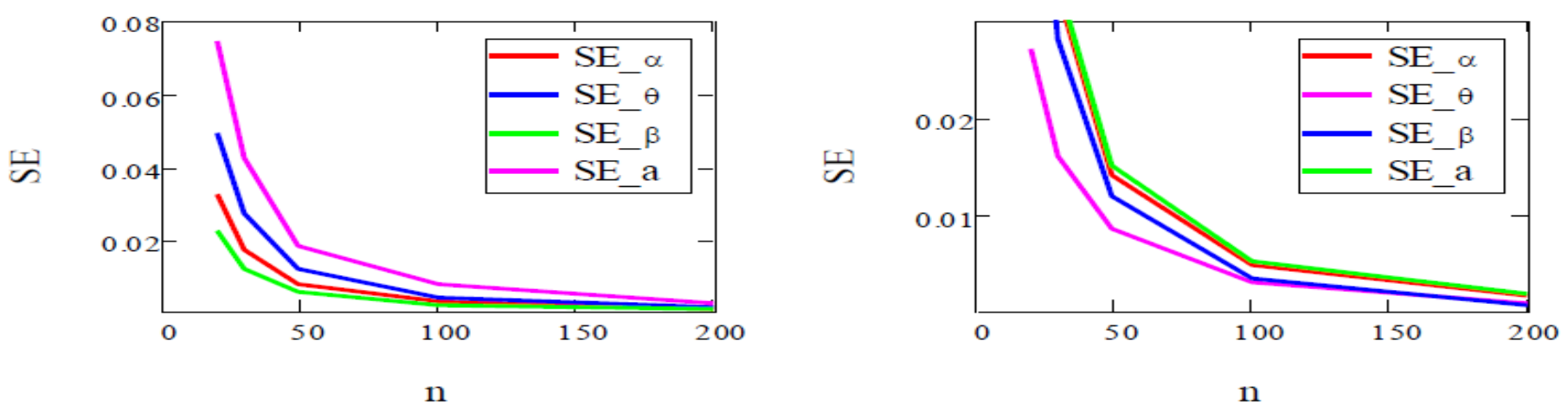

Figure 6: Standard errors of $\alpha, \beta, \theta$ and a for set VI and set II

\section{Data Analysis}

In this section, three real data sets are analyzed to illustrate the merit of EWPF distribution compared with some other models; namely, Weibull power function (WPF), beta Weibull (BW), beta modified Weibull (BMW) (see Silva et al. (2010)), exponentiated generalized modified Weibull (EGMW) (see Aryal and Elbatal (2015)), Kumerswmay power function (KPF), PF and transmuated power function ( TPF).

We obtain the MLE and their corresponding standard errors (in parentheses) of the model parameters. To compare the distribution models, we consider criteria like; KolmogorovSmirnov (K-S) statistic, Akaike information criterion (AIC), the correct Akaike information criterion (CAIC), Bayesian information criterion (BIC) and Hannan-Quinn information criterion (HQIC). However, the better distribution corresponds to the smaller values of AIC BIC, CAIC, HQIC criteria and K-S statistic. Further, we plot the histogram and empirical cdfs for each data set and the estimated pdf of the EWPF, WPF, BMW, BW, EGMW, KPF, PF and TPF models.

\subsection{Data Set 1: Acute Myelogenous data}

The first data were first analyzed by Feigl and Zelen (1965). The data represent the survival times, in weeks, of 33 patients suffering from Acute Myelogenous Leukaemia. The data are: 65, 156, 100, 134, 16, 108, 121, 4, 39, 143, 56, 26, 22, 1, 1, 5, 65, 56, 65, 17, 7, 16, 22, 3, 4, 2, 3,8, $4,3,30,4,43$.

Table 1 lists the values of MLEs of parameters and their standard errors (SE) in parenthesis. Table 2 lists the values of AIC, CAIC, BIC, HQIC and K-S. 


\begin{tabular}{|c|c|c|c|c|c|c|c|c|}
\hline \multirow{2}{*}{ Models } & \multicolumn{8}{|c|}{ Estimated Parameters } \\
\hline & a & $\alpha$ & $\beta$ & & & $b$ & & \\
\hline$E W P F$ & $\begin{array}{c}63.968 \\
(0.0042)\end{array}$ & $\begin{array}{c}6.082 \\
(0.376)\end{array}$ & $\begin{array}{l}0.083 \\
(0.01)\end{array}$ & 156 & $\begin{array}{c}1.681 \\
(0.000088)\end{array}$ & - & - & - \\
\hline$B M W$ & $\begin{array}{c}0.079 \\
(0.1)\end{array}$ & $\begin{array}{c}37.15 \\
(46.903)\end{array}$ & & $\begin{array}{c}0.00006 \\
(0.003)\end{array}$ & & $\begin{array}{c}0.883 \\
(0.177)\end{array}$ & $\begin{array}{c}0.001 \\
(0.00014)\end{array}$ & \\
\hline$E G M W$ & - & $\begin{array}{c}0.229 \\
(0.008)\end{array}$ & $\begin{array}{c}0.678 \\
(0.0003)\end{array}$ & $\begin{array}{c}1 \\
(0.016)\end{array}$ & $\begin{array}{c}0.082 \\
(0.153)\end{array}$ & - & - & $\begin{array}{l}.00031 \\
(4.838) \\
\end{array}$ \\
\hline$W P F$ & $\begin{array}{c}2.681 \\
(0.631)\end{array}$ & 156 & $\begin{array}{c}4.391 \\
(2.377)\end{array}$ & & $\begin{array}{c}4.391 \\
(2.377) \\
\end{array}$ & $\begin{array}{c}0.16 \\
(0.076)\end{array}$ & & \\
\hline$P F$ & - & $\begin{array}{c}0.449 \\
(0.078) \\
\end{array}$ & - & 156 & - & - & - & - \\
\hline KP & $\begin{array}{c}0.5 \\
(0.1180) \\
\end{array}$ & $\begin{array}{c}0.392 \\
(0.124)\end{array}$ & - & 156 & - & $\begin{array}{c}0.392 \\
(0.124) \\
\end{array}$ & - & - \\
\hline$T P F$ & - & $\begin{array}{c}0.817 \\
(0.108)\end{array}$ & 156 & - & $\begin{array}{c}1.194 \\
(0.064)\end{array}$ & - & - & - \\
\hline
\end{tabular}

\begin{tabular}{|c|c|c|c|c|c|}
\hline Models & $\boldsymbol{A I C}$ & $\boldsymbol{B I C}$ & $\boldsymbol{C A I C}$ & $\boldsymbol{H Q I C}$ & $\boldsymbol{K}-\boldsymbol{S}$ \\
\hline $\boldsymbol{E W P F}$ & 305.852 & 313.335 & 308.074 & 308.37 & 0.1199 \\
\hline $\boldsymbol{B M W}$ & 318.967 & 326.449 & 321.189 & 321.484 & 0.939 \\
\hline $\boldsymbol{E} \boldsymbol{G M W}$ & 317.303 & 324.786 & 319.525 & 318.821 & 0.138 \\
\hline $\boldsymbol{W P F}$ & 307.804 & 313.79 & 309.232 & 309.818 & 0.1214 \\
\hline $\boldsymbol{P F}$ & 965.418 & 968.411 & 965.818 & 966.425 & 0.145 \\
\hline $\boldsymbol{K P}$ & 329.734 & 335.72 & 331.162 & 331.748 & 0.2645 \\
\hline $\boldsymbol{T P F}$ & 335.131 & 339.62 & 335.959 & 336.642 & 0.183 \\
\hline
\end{tabular}

Table 1: MLEs and their SEs (in parentheses) for Acute Myelogenous data

Table 2: Statistics measures for Acute Myelogenous data

It is clear from Table 2 that the EWPF distribution provides a better fit than the other competitive models. It has the the smallest values for K-S, AIC, CAIC, BIC and HQIC among those considered here. Plots of the fitted densities and the histogram are given in Figure 7. 


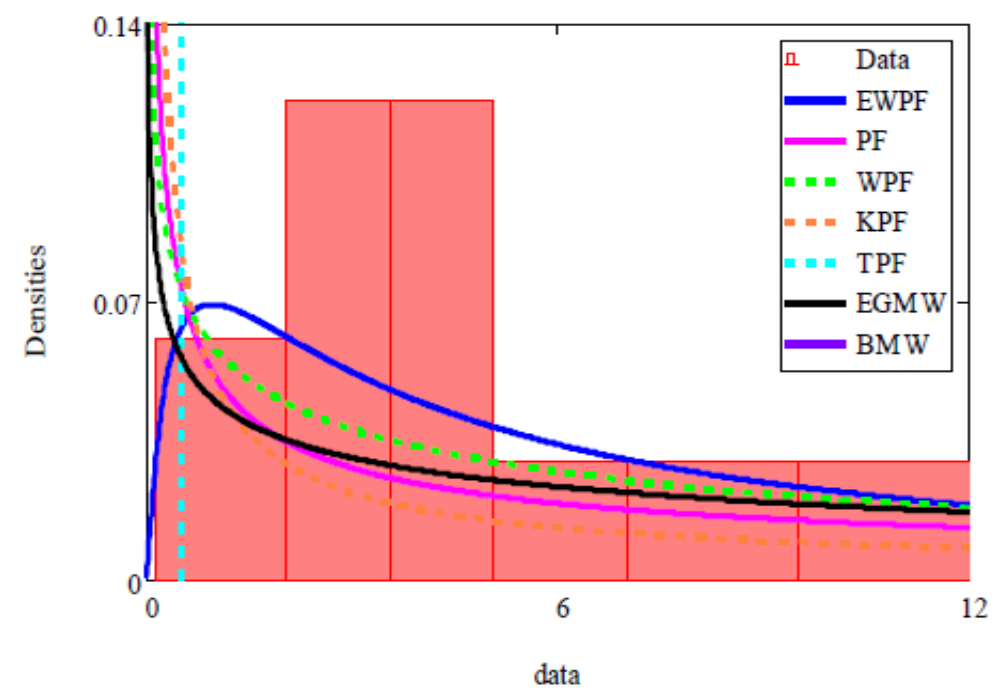

(a) Estimated pdfs

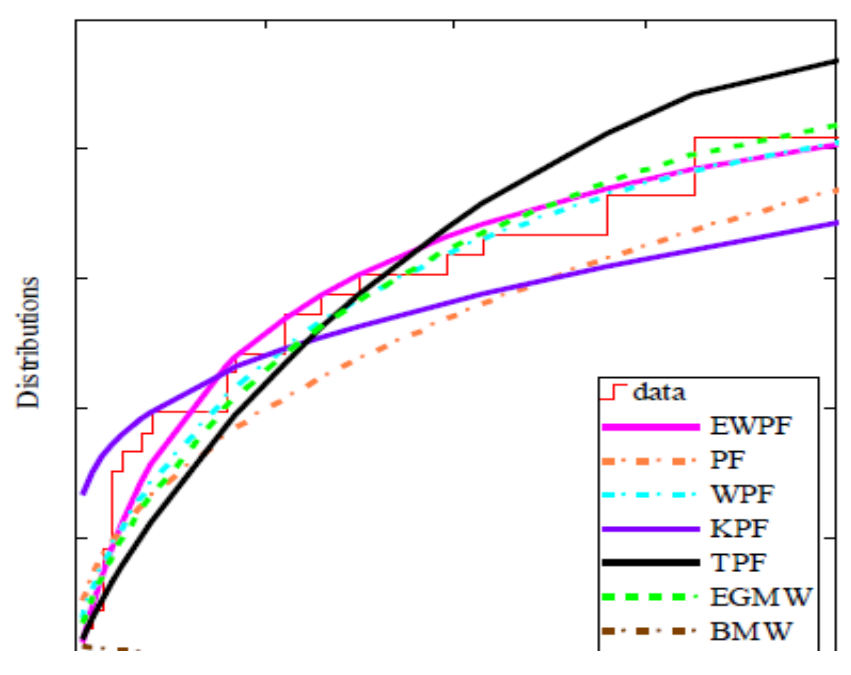

(b) Estimated cdfs

Figure 7. Plots of the estimated pdfs and cdfs for the EWPF, BMW, EGMW, WPF, KPF, PF and TPF models for Acute Myelogenous data

\subsection{Data Set 2: Actual Taxes data}

The second real data have been used by Nassar and Nada (2011). The data represent the monthly actual taxes revenue in Egypt from January 2006 to November 2010. The distribution is highly skewed to the right. The actual taxes revenue data (in 1000 million Egyptian pounds) are: $5.9,20.4,14.9,16.2,17.2,7.8,6.1,9.2,10.2,9.6,13.3,8.5,21.6,18.5,5.1,6.7,17,8.6,9.7$, $39.2,35.7,15.7,9.7,10,4.1,36,8.5,8,9.2,26.2,21.9,16.7,21.3,35.4,14.3,8.5,10.6,19.1$, $20.5,7.1,7.7,18.1,16.5,11.9,7,8.6,12.5,10.3,11.2,6.1,8.4,11,11.6,11.9,5.2,6.8,8.9,7.1$, 10.8 . 
Table 3: MLEs and their SEs (in parentheses) for the Actual Taxes data

\begin{tabular}{|c|c|c|c|c|c|c|c|}
\hline \multirow{2}{*}{ Models } & \multicolumn{7}{|c|}{ Estimated Parameters } \\
\hline & a & $\alpha$ & $\beta$ & & & $b$ & \\
\hline$E W P F$ & $\begin{array}{c}63.574 \\
(0.00455)\end{array}$ & $\begin{array}{c}8.364 \\
(0.5902)\end{array}$ & $\begin{array}{c}0.066 \\
(0.00621)\end{array}$ & 39.2 & $\begin{array}{c}7.377 \\
(0.00019)\end{array}$ & - & - \\
\hline$B \boldsymbol{W}$ & $\begin{array}{c}55.574 \\
(42.263)\end{array}$ & $\begin{array}{c}1.391 \\
(0.403)\end{array}$ & - & - & - & $\begin{array}{c}0.031 \\
(0.00561)\end{array}$ & $\begin{array}{c}1.238 \\
(0.222)\end{array}$ \\
\hline$W P F$ & $\begin{array}{c}5.666 \\
(1.036) \\
\end{array}$ & - & 39.2 & - & $\begin{array}{c}15.321 \\
(10.509) \\
\end{array}$ & $\begin{array}{c}0.118 \\
(0.078) \\
\end{array}$ & - \\
\hline$P F$ & - & $\begin{array}{c}0.81 \\
(0.1055)\end{array}$ & - & 39.2 & - & - & - \\
\hline KPF & $\begin{array}{c}0.5 \\
(0.09934) \\
\end{array}$ & $\begin{array}{c}0.44 \\
(0.104) \\
\end{array}$ & - & 39.2 & - & $\begin{array}{c}0.44 \\
(0.104) \\
\end{array}$ & - \\
\hline$T P F$ & - & $\begin{array}{c}1.172 \\
(0.1194)\end{array}$ & - & 39.2 & $\begin{array}{c}0.962 \\
(0.093)\end{array}$ & - & - \\
\hline
\end{tabular}

Table 4: Statistics measures for the Actual Taxes data.

\begin{tabular}{|c|c|c|c|c|c|}
\hline Models & $\boldsymbol{A I C}$ & $\boldsymbol{B I C}$ & $\boldsymbol{C A I C}$ & $\boldsymbol{H Q I C}$ & $\boldsymbol{K}-\boldsymbol{S}$ \\
\hline $\boldsymbol{E W P F}$ & 383.784 & 394.172 & 384.916 & 387.84 & 0.08234 \\
\hline $\boldsymbol{B W}$ & 401.277 & 409.588 & 402.018 & 404.521 & 5.212 \\
\hline $\boldsymbol{W P F}$ & 401.037 & 409.347 & 401.778 & 404.28 & 0.14317 \\
\hline $\boldsymbol{P F}$ & 12600 & 126600 & 126200 & 126300 & 0.304 \\
\hline $\boldsymbol{K P F}$ & 499.825 & 508.135 & 500.565 & 503.06 & 0.4087 \\
\hline $\boldsymbol{T P F}$ & 417.729 & 423.961 & 418.165 & 420.16 & 0.195 \\
\hline
\end{tabular}

It is clear from Table 4 that the EWPF distribution provides a better fit than the other comptitive models. It has the the smallest K-S, AIC, CAIC, BIC and HQIC values among those considered here. Plots of the fitted densities and the histogram are given in Figure 8. 


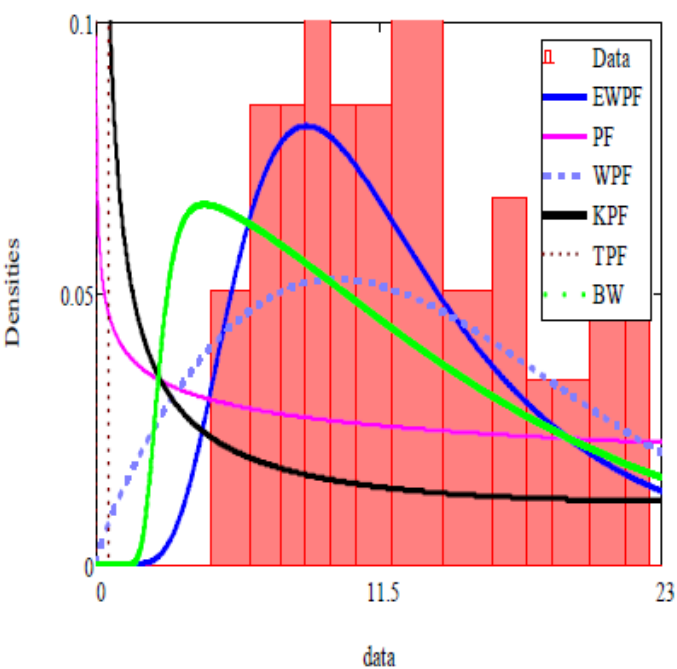

(a) Estimated pdfs

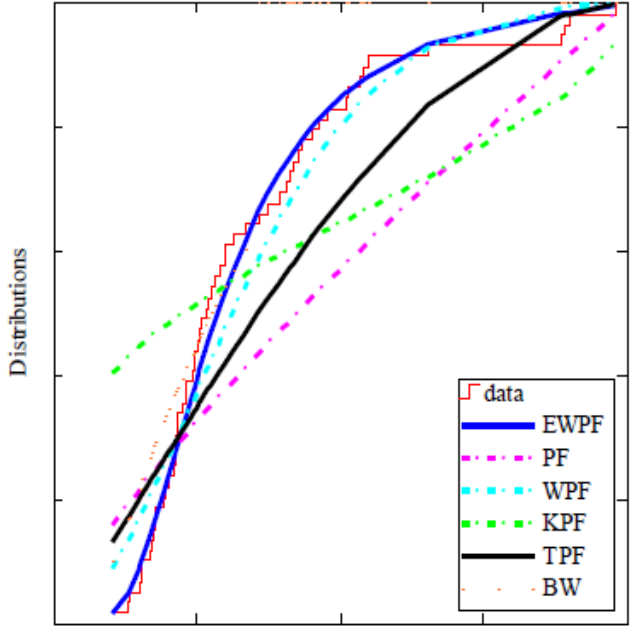

(b) Estimated cdfs

Figure 8. Plots of the estimated pdfs and cdfs for the EWPF, BW, WPF, KPF, PF and TPF models for the Actual Taxes data

\subsection{Data set 3: Failure Time Data}

The third data set are provided in Murthy et al. (2004) about time between failures for 30 repairable items. The data are listed as the following: 1.43, 0.11, 0.71, 0.77, 2.63, 1.49, 3.46, $2.46,0.59,0.74,1.23,0.94,4.36,0.40,1.74,4.73,2.23,0.45,0.70,1.06,1.46,0.30,1.82,2.37$, $0.63,1.23,1.24,1.97,1.86,1.17$.

Table 5: MLEs and their SEs (in parentheses) for the failure time data

\begin{tabular}{|c|c|c|c|c|c|c|c|}
\hline \multirow{2}{*}{ Models } & \multicolumn{7}{|c|}{ Estimated Parameters } \\
\cline { 2 - 8 } & $\mathrm{a}$ & $\alpha$ & $\beta$ & & & $b$ & \\
\hline \multirow{2}{*}{$\boldsymbol{W P F}$} & $\begin{array}{c}39.68 \\
(0.02)\end{array}$ & $\begin{array}{c}5.629 \\
(0.3977)\end{array}$ & $\begin{array}{c}0.248 \\
(0.032)\end{array}$ & 4.73 & $\begin{array}{c}1.033 \\
(0.00012)\end{array}$ & & \\
\hline $\boldsymbol{B} \boldsymbol{B} \boldsymbol{W}$ & $\begin{array}{c}0.539 \\
(0.934)\end{array}$ & $\begin{array}{c}50.552 \\
(112.102)\end{array}$ & - & $\begin{array}{c}0.274 \\
(0.189)\end{array}$ & - & $\begin{array}{c}772 \\
(0.016)\end{array}$ & $\begin{array}{c}0.857 \\
(0.333)\end{array}$ \\
\hline $\boldsymbol{W P F}$ & $\begin{array}{c}5.636 \\
(1.5679)\end{array}$ & 4.73 & - & - & $\begin{array}{c}6.539 \\
(6.845)\end{array}$ & $\begin{array}{c}0.22 \\
(0.221)\end{array}$ & - \\
\hline $\boldsymbol{P F}$ & - & $\begin{array}{c}0.631 \\
(0.1151)\end{array}$ & - & 4.73 & - & & \\
\hline $\boldsymbol{K} \boldsymbol{K P F}$ & $\begin{array}{c}1 \\
(0.2939)\end{array}$ & $\begin{array}{c}0.646 \\
(0.177)\end{array}$ & - & 4.73 & - & $\begin{array}{c}0.646 \\
(0.177)\end{array}$ & - \\
\hline $\boldsymbol{T P F}$ & - & $\begin{array}{c}1.075 \\
(0.1637)\end{array}$ & - & 4.73 & $\begin{array}{c}1.44 \\
(0.209)\end{array}$ & & \\
\hline
\end{tabular}


Amal S. Hassan ${ }^{1}$, Salwa M. Assar ${ }^{2}$

Table 6: Statistics measures for the failure time data

\begin{tabular}{|c|c|c|c|c|c|}
\hline Model & AIC & BIC & CAIC & HQIC & K-S \\
\hline $\boldsymbol{E W P F}$ & 89.248 & 96.254 & 91.748 & 91.489 & 0.07328 \\
\hline $\boldsymbol{B M W}$ & 94.406 & 101.412 & 96.906 & 96.647 & 0.86667 \\
\hline $\boldsymbol{W P F}$ & 92.324 & 97.929 & 93.924 & 94.117 & 0.07705 \\
\hline $\boldsymbol{P F}$ & 445.571 & 448.373 & 446.015 & 446.46 & 0.288 \\
\hline $\boldsymbol{K P F}$ & 109.747 & 115.352 & 111.347 & 111.54 & 0.26293 \\
\hline $\boldsymbol{T P F}$ & 101.458 & 105.662 & 102.381 & 102.80 & 0.139 \\
\hline
\end{tabular}

As shown in Table 6; the EWPF distribution provides a better fit than the other competitive models, since it has the the smallest K-S, AIC, CAIC, BIC and HQIC values. Plots of the fitted densities and the histogram are given in Figure 9.

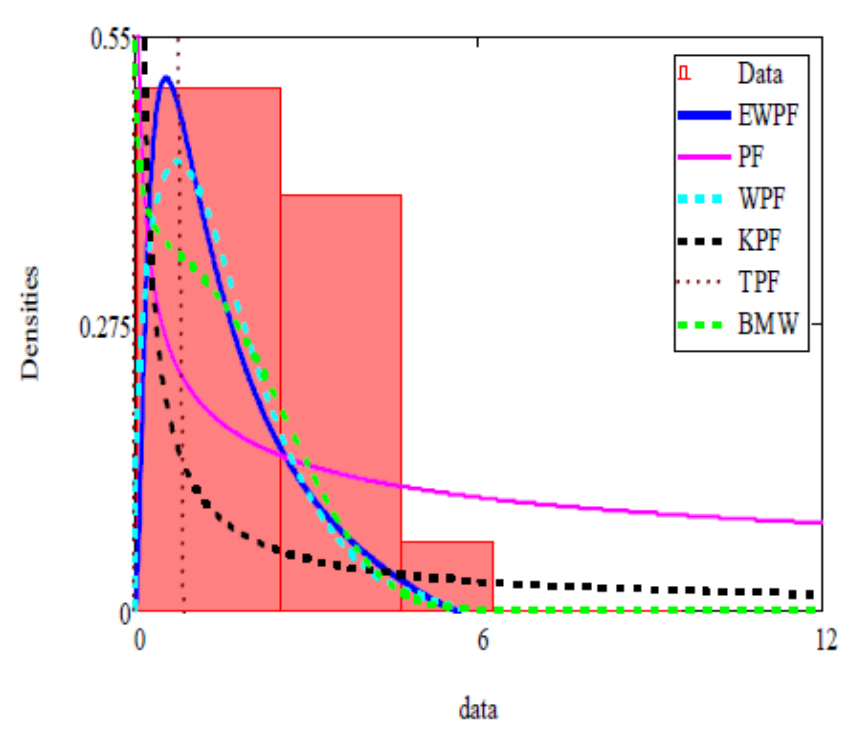

(a) Estimated pdfs

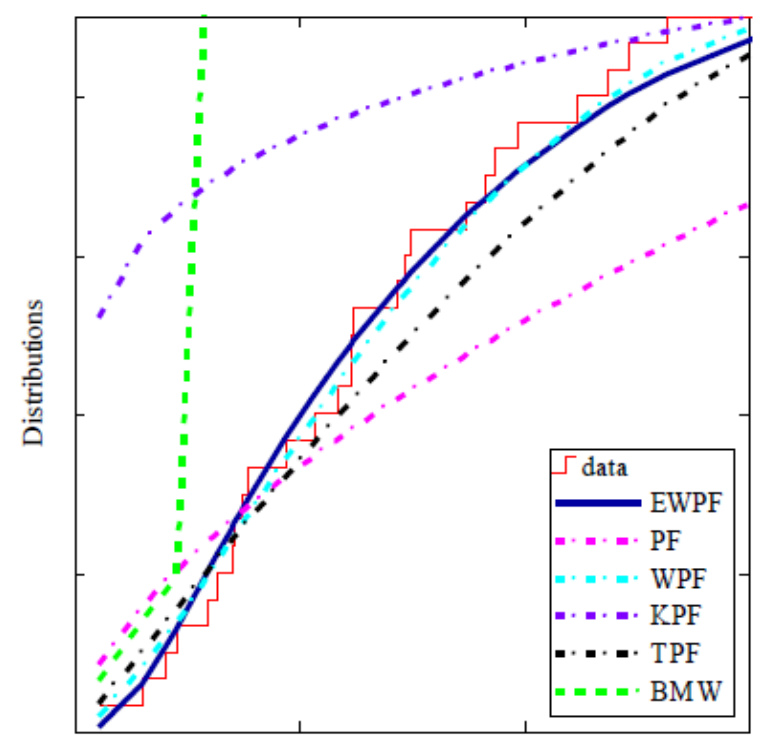

(b) Estimated cdfs

Figure 9. Plots of the estimated pdfs and cdfs for the EWPF, BMW, WPF, KPF, PF and TPF models for failure time data

\section{Concluding Remarks}

In this paper, a new five-parameter, called the exponentiated Weibull power function distribution is introduced based on exponentiated Weibull-G family. The exponentiated Weibull power function distribution includes the Weibull power function distribution presented by Tahir et al. (2016 a). Some mathematical properties are derived. The maximum likelihood method is employed for estimating the model parameters. A simulation study is presented to evaluate the maximum likelihood estimates for model parameters. The practical importance of the EWPF distribution was demonstrated in three applications to show superior performance in comparison with several other former lifetime distributions. Applications showed that the 
EWPF model can be performed better than the Weibull power function, power function, Kumerswmay power function, beta Weibull, beta modified Weibull, exponentiated generalized modified Weibull and the transmuted power function distributions.

\section{References}

[1] Afify, A.Z., Cordeiro, G.M., Yousof, H. M., Alzaatreh, A. and Nofal, Z.M. (2016). The Kumaraswamy transmuted-G family of distributions: Properties and applications. Journal of Data Science, 14, 245-270.

[2] Ahsanullah, M. and Lutful-Kabir, A.B.M. (1974). A characterization of the power function distribution. The Canadian Journal of Statistics, 2, 95-98.

[3] Alexander, C., Cordeiro, G.M., Ortega, E.M.M., and Sarabia, J.M. (2012). Generalized beta generated distributions. Computational Statistics and Data Analysis, 56, 1880-1897.

[4] Alizadeh, M., Rasekhi, M., Yousof, H. M., and Hamedani, G. G. (2016). The transmuted Weibull G family of distributions. Hacettepe Journal of Mathematics and Statistics. Doi: 10.15672/HJMS.2017.440.

[5] Alzaatreh, A., Lee, C. and Famoye, F. (2013). A new method for generating families of continuous distributions. Metron, 71, 63-79.

[6] Aryal, G. and Elbatal, I. (2015). On the exponentiated generalized modified Weibull distribution. Communications for Statistical Applications and Methods, 22(4), 333-348.

[7] Bourguignon, M., Silva, R.B., and Cordeiro, G.M. (2014). The Weibull-G family of probability distributions. Journal of Data Science, 12, 53-68.

[8] Bursa, N. and Kadilar, G. O. (2017). The exponentiated Kumaraswamy power function distribution. Hacettepe University Bulletin of Natural Sciences and Engineering Series Mathematics and Statistics, 46(2), 1 - 19.

[9] Chakraborty, S. and Handique, L. (2017). The generalized Marshall-OlkinKumaraswamy-G family of distributions. Journal of Data Science, 15, 391-422.

[10]Chang, S.K. (2007). Characterization of the power function by the independence of record values. Journal of the Chungcheong Mathematical Society, 20,140-145.

[11]Cordeiro, G.M. and Brito, R.S. (2012). The beta power distribution. Brazilian Journal of Probability and Statistics, 26, 88-112.

[12]Cordeiro G.M. and de Castro M. (2011). A new family of generalized distributions. Journal of Statistical Computation and Simulation, 81, 883-893. 
[13]Cordeiro, G. M., Ortega, E. M. M., \& Cunha, D. C. C. (2013). The exponentiated generalized class of distributions. Journal of Data Science, 11, 1-27.

[14]Cordeiro, G. M., Ortega, M. M. E. and Ramires, T. G. (2015). A new generalized Weibull family of distributions: mathematical properties and applications, Journal of Statistical Distributions and Applications, 2(13), 1-26.

[15]Dallas, A. C. (1976). Characterization of Pareto and power function distribution. Annals of the Institute of Statistical Mathematics, 28(1), 491-497.

[16]Eugene, N., Lee C., and Famoye, F. (2002). Beta-normal distribution and its applications. Communication in Statistics - Theory Methods, 31, 497-512.

[17]Feigl, P. and Zelen, M. (1965). Estimation of exponential probabilities with concomitant information. Biometrics, 21, 826-838.

[18] Greenwood, J.A., Landwehr, J.M., and Matalas, N.C. (1979). Probability weighted moments: Definitions and relations of parameters of several distributions expressible in inverse form. Water Resources Research, 15, 1049-1054.

[19]Haq, M. A., Butt, N. S., Usman, R. M., Fattah, A. A. (2016). Transmuted power function distribution. Gazi University Journal of Science, 29 (1), 177-185.

[20]Hassan, A. S. and Elgarhy, M., (2016 a). Kumaraswamy Weibull-generated family of distributions with applications. Advances and Applications in Statistics, 48, 205-239.

[21]Hassan, A. S. and Elgarhy, M. (2016 b). A new family of exponentiated Weibullgenerated distributions. International Journal of Mathematics And its Applications, 4, 135-148.

[22] Hassan, A. S., and Hemeda, S. E. (2016). The additive Weibull-g family of probability distributions. International Journals of Mathematics and Its Applications, 4, 151-164.

[23] Hassan, A. S., Hemeda, S. E., Maiti, S., and Pramanik, S., (2017). The generalized ddditive Weibull-G family of distributions. International Journal of Statistics and Probability, 6(5), 65-83.

[24]Jones, M.C. (2004). Families of distributions arising from the distributions of order statistics. Test, 13, 1-43.

[25] Kenney, J. and Keeping, E. (1962). Mathematics of Statistics, Volume 1, Third edition, Van Nostrand, Princeton. 
[26]Meniconi, B. and Barry, M. D. (1996). The power function distribution: a useful and simple distribution to assess electrical component reliability. Microelectronics Reliability, 36(9), 1207-1212.

[27] Moors, J. J. A. (1988). A quantile alternative for kurtosis. Journal of the Royal Statistical Society. Series D (The Statistician), 37(1), 25-32.

[28] Murthy, D.N.P., Xie, M. and Jiang, R. (2004). Weibull models, Series in Probability and Statistics, John Wiley, New Jersey.

[29] Nassar, M. M. and Nada, N. K. (2011). The beta generalized Pareto distribution. Journal of Statistics: Advances in Theory and Applications, 6, 1-17.

[30] Oguntunde, P., Odetunmibi, O. A., Okagbue, H. I. Babatunde, O. S. and Ugwoke, P. O. (2015). The Kumaraswamy-power distribution: A generalization of the power distribution. International Journal of Mathematical Analysis, 9(13), 637-645.

[31]Risti'c, M.M., and Balakrishnan, N. (2012). The gamma-exponentiated exponential distribution. Journal of Statistical Computation and Simulation, 82,1191-1206.

[32] Silva, G. O., Ortega, E. M. M. and Cordeiro, G. M. (2010). The beta modified Weibull distribution. Lifetime Data Analysis, 16(3), 409-430.

[33]Tahir, M., Alizadeh, M., Mansoor, M., Cordeiro, G. M. and Zubair, M. (2016 a). The Weibull-power function distribution with applications. Hacettepe University Bulletin of Natural Sciences and Engineering Series Mathematics and Statistics, 45(1), 245 - 265.

[34]Tahir, M., Zubair, M., Mansoor, M., Cordeiro, G. M, Alizadeh, M., and Hamedani, G. G. (2016b). A new Weibull-G family of distributions. Hacettepe Journal of Mathematics and Statistics, 45 (2), $629-647$.

[35] Tavangar, M. (2011). Power function distribution characterized by dual generalized order statistics. Journal of the Iranian Statistical Society, 10, 13-27.

[36] Yousof, H. M., Rasekhi, M., Afify, A. Z., Ghosh, I., Alizadeh, M., and Hamedani, G. G. (2017). The beta Weibull-G family of distributions: Theory, characterizations and applications. Pakistan Journal of Statistics, 33(2), 95-116.

[37]Zenga, M, (2007). Inequality curve and inequality index based on the ratios between lower and upper arithmetic means. Statistica e Applicazioni, 4, 3-27.

[38]Zografos, K., and Balakrishnan, N. (2009). On families of beta- and generalized gammagenerated distributions and associated inference. Statistical Methodology, 6, 344-362. 
${ }^{1}$ Amal S. Hassan

Department of Mathematical Statistics, Cairo University Institute of Statistical Studies \& Research, Egypt.

E-mail ID: dr.amalelmoslamy@ gmail.com

${ }^{2}$ Salwa M. Assar

Department of Mathematical Statistics, Cairo University

Institute of Statistical Studies \& Research, Egypt.

E-mail ID: salwaassar@yahoo.com 
